\title{
COBORDISM FOR POINCARÉ DUALITY GROUPS
}

\author{
BY ROBERT BIERI AND BENO ECKMANN
}

Communicated by S. Eilenberg, October 9, 1975

1. Relative homology for pairs. Homology and cohomology for a pair of groups $G \supset S$ (cf. [6]) can be extended to pairs $(G, S)$ consisting of a group $G$ and a family of subgroups $S=\left\{S_{i}\right\}$, as follows: If $S=\varnothing$ one takes the usual (absolute) groups of $G$. If $S \neq \varnothing$, let $\Delta$ be the kernel of the $G$-homomorphism $\bigoplus_{i} \mathrm{Z}\left(G / S_{i}\right) \rightarrow \mathrm{Z}$ given by augmentations; $A$ being a $G$-module, we put $H^{k}(G, S ; A)=H^{k-1}(G ; \operatorname{Hom}(\Delta, A))$ and $H_{k}(G, S ; A)=H_{k-1}(G ; \Delta \otimes A)$ where $G$ acts diagonally in $\operatorname{Hom}(\Delta, A)$ and $\Delta \otimes A$. One has exact sequences

$$
\begin{aligned}
& \cdots \rightarrow H^{k}(G, S ; A) \rightarrow H^{k}(G ; A) \rightarrow \prod_{i} H^{k}\left(S_{i} ; A\right) \stackrel{\delta}{\longrightarrow} H^{k+1}(G, S ; A) \rightarrow \cdots, \\
& \cdots \rightarrow H_{k+1}(G, S ; A) \stackrel{\partial}{\longrightarrow} \bigoplus_{i} H_{k}\left(S_{i} ; A\right) \rightarrow H_{k}(G ; A) \rightarrow H_{k}(G, S ; A) \rightarrow \cdots
\end{aligned}
$$

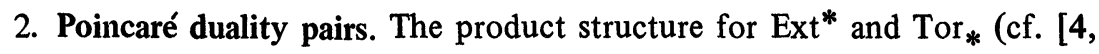
Chapter XI]) yields, for $\alpha \in H_{n}(G, S ; B)$, cap-products $\alpha \cap-: H^{k}(G ; A) \rightarrow$ $H_{n-k}(G, S ; B \otimes A)$ and $H^{k}(G, S ; A) \rightarrow H_{n-k}(G ; B \otimes A)$, with diagonal $G$-action in $B \otimes A$.

(1) Definition. $(G, S)$ is a Poincaré duality pair of dimension $n$ (in short: $P D^{n}$-pair) if there is a $G$-module structure $\widetilde{\mathbf{Z}}$ on $\mathbf{Z}$ and an element $e \in$ $H_{n}(G, S ; \widetilde{\mathbf{Z}})$ such that $e \cap-: H^{k}(G ; A) \rightarrow H_{n-k}(G, S ; \widetilde{A})$ is an isomorphism for all $A$ and $k$.

$\widetilde{\mathbf{Z}}$ is called the "dualizing" module. $\widetilde{A}$ stands for $\widetilde{\mathbf{Z}} \otimes A$. If $S$ is empty, (1) coincides with the usual definition (cf. [1]) of a Poincaré duality group of dimension $n$ (in short: $P D^{n}$-group).

(2) TheOREM. $(G, S)$ is a $P D^{n}$-pair with dualizing module $\widetilde{\mathbf{Z}}$ if and only if $G$ is a duality group (cf. [3]) with dualizing module. $\widetilde{\Delta}$.

Thus various results and criteria for duality groups can be applied to $P D^{n}$. pairs; note that $\widetilde{\Delta}$ is $\mathbf{Z}$-free. We give here, and in $\S 3$, a list of consequences of (1): Definition (1) is equivalent with $e \cap-: H^{k}(G, S ; A) \rightarrow H_{n-k}(G ; \widetilde{A})$ being an isomorphism for all $A$ and $k$. The module $\widetilde{\mathbf{Z}}$ and the dimension $n$ are determined by the pair $(G, S)$. The pair is called orientable if $G$ acts trivially on $\widetilde{Z}$,

AMS (MOS) subject classifications (1970). Primary 18H10, 57B10; Secondary 20J05, $57 \mathrm{A70}, 57 \mathrm{C} 20$.

Key words and phrases. Relative homology of groups, Poincaré duality, oriented cobordism, nonoriented cobordism, graph of groups, finite projective resolutions, signature, characteristic classes. 
otherwise nonorientable. The group $H_{n}(G, S ; \widetilde{\mathbf{Z}})$ is infinite cyclic generated by $e$; choice of a generator $e$ is called an orientation (even in the nonorientable case!). The family $S$ is finite; all $S_{t}$ in $S$ are $P D^{n-1}$-groups; an orientation of $(G, S)$ determines an orientation for each $S_{i}$. We call $S$ (with these orientations) the boundary of the (oriented) $P D^{n}$-pair $(G, S)$. For an oriented group the minus sign denotes change of orientation.

3. Extensions and amalgams. Using (2) and criteria for duality one proves

(3) THEOREM. Let $N \succ G \stackrel{\pi}{\longrightarrow} Q$ be a short exact sequence of groups, $S$ a family of subgroups $S_{i}$ of $G$ each containing $N$, and $K=\pi(S)$. If $N$ is a $P D^{n}$-group and $(Q, K)$ is a $P D^{m}$-pair, then $(G, S)$ is a $P D^{n+m}$-pair.

There is a converse of this in the sense of [2, Theorem A]; and there is also a "finite extension Theorem" generalizing the corresponding result in [1] and [5].

(4) THEOREM. (a) If the subgroups $U$ of $G$ and $V$ of $H$ are isomorphic, and if $(G, S \cup U)$ and $(H, R \cup V)$ are $P D^{n}$-pairs then so is $\left(G{ }^{*} H, S \cup R\right){ }^{1}$

(b) Let $U, V$ be isomorphic subgroups of $G$, with $\sigma: U \stackrel{U \cong}{\Longrightarrow}$. If $(G, S \cup U \cup V)$ is a $P D^{n}$-pair, then so is $\left(G_{* U, \sigma}, S\right)$, where $G *_{U, \sigma}$ is the $H N N$-extension for $\sigma$.

Repeated application of (4) yields, for the "fundamental group $G(\mathbb{S})$ of a graph of groups" in the sense of Serre [7]:

(5) THEOREM. Let $\left(G^{\nu}, S^{\nu}\right)$ be a finite family of $P D^{n}$-pairs, and $($ a graph of groups whose vertices $V(\circlearrowleft)$ are the groups $G_{\nu}$ and whose edges $E(\circlearrowleft)$ some pairs of isomorphic subgroups $S_{i}^{(\nu)}, S_{i}^{\nu^{\prime}} \in \bigcup_{\nu} S^{(\nu)}$. Further, let $S$ be the family of those $S_{i}^{(\nu)}$ not occurring in $E(\circlearrowleft)$. Then $(G(\mathcal{S}), S)$ is a $P D^{n}$-pair.

4. Oriented cobordism. Here all $P D$-groups and -pairs are assumed orientable and oriented.

(6) Definition. Two finite families $S, T$ of $P D^{n-1}$-groups are cobordant if there exist finitely many $P D^{n}$-pairs such that $S \cup(-T)$ is the disjoint union of their boundaries.

This is an equivalence relation: it is easily seen that it is reflexive and symmetric; transitivity is proved by means of (5). We write $\langle S\rangle$ for the class of $S$, and $\Omega_{n}$ for the set of all classes of families $S$ of $P D^{n}$-groups. With addition of classes defined by $\langle S\rangle+\langle T\rangle=\langle S \cup T\rangle, \Omega_{n}$ is an Abelian group $(0=\varnothing$, $-\langle S\rangle=\langle-S\rangle)$; it is generated by the classes $\langle S\rangle$ of single groups. $\Omega_{0} \cong \mathbf{Z}$, generated by the class $\langle 1\rangle$ of the trivial group. The direct product of groups defines a graded (skew-) commutative multiplication $\Omega_{n} \times \Omega_{m} \rightarrow \Omega_{n+m}$ turning the graded group $\Omega_{*}$ into a graded ring with unit.

\footnotetext{
${ }^{1}$ For families $S, T$, the union $S \cup T$ is taken with respect to the disjoint union of the indexing sets.
} 
The signature of an orientable $P D^{n}$-group is defined as for manifolds (for dimensions $4 k$, otherwise 0 ). One can show that it is 0 for $\langle S\rangle=0$, and that it defines a unitary ring homomorphism $\Omega_{*} \rightarrow \mathbf{Z}$. From known examples of $P D^{4}$-groups with signature $\neq 0$ (even with arbitrarily large signature) it follows that $\Omega_{*}$ does not consist of $\Omega_{0}$ only.

Oriented cobordism can, of course, also be defined by admitting orientable and nonorientable $P D^{n}$-groups and -pairs. Using (4)(a) above, one proves that the parity of the Euler characteristic is an invariant. Nonorientiable higher genus surfaces with odd Euler characteristic thus provide examples of $P D^{2}$-groups $S$ with $\langle S\rangle \neq 0$.

5. Nonoriented cobordism. If orientable and nonorientable $P D^{n}$-groups and -pairs are admitted and all orientations disregarded, the nonoriented cobordism ring $\mathfrak{N}_{*}$ is obtained (all elements are of order 2 ). One then can prove that for $\langle S\rangle=0$ all Stiefel-Whitney numbers of $S$ are $0(\bmod 2$ characteristic classes for $P D^{n}$-groups and -pairs can be defined algebraically via the Steenrod squares and the $\mathrm{Wu}$ formula).

\section{REFERENCES}

1. R. Bieri, Gruppen mit Poincaré-Dualitat, Comment. Math. Helv. 47 (1972), 373396.

2. - Normal subgroups in duality groups and in groups of cohomological dimension 2, J. Pure Appl. Algebra (to appear).

3. R. Bieri and B. Eckmann, Groups with homological duality generalizing Poincaré duality, Invent. Math. 20 (1973), 103-124. MR 49 \#5204.

4. H. Cartan and S. Eilenberg, Homological algebra, Princeton Univ. Press, Princeton, N. J., 1956. MR 17, 1040.

5. F. E. A. Johnson and C. T. C. Wall, On groups satisfying Poincaré duality, Ann. of Math. (2) 96 (1972), 592-598. MR 47 \#358.

6. L. Ribes, On a cohomology theory for pairs of groups, Proc. Amer. Math. Soc. 21 (1969), 230-234. MR 38 \#4563.

7. J.-P. Serre, Arbres, amalgames et $S L_{2}$, Lecture Notes in Math., Springer-Verlag, Berlin and New York (to appear).

UNIVERSITÄT FREIBURG I. BR., BRD AND EIDGEN. TECHNISCHE HOCHSCHULE, ZÜRICH, SWITZERLAND 